\title{
Characterization of a rat model of moderate liver dysfunction based on alpha-naphthylisothiocyanate-induced cholestasis
}

\author{
Melanie K. Bothe ${ }^{1}$, Christoph Meyer ${ }^{1}$, Udo Mueller², Jean-Christophe Queudot ${ }^{3}$, \\ Virginie Roger ${ }^{4}$, Johannes Harleman ${ }^{1}$ and Martin Westphal1 \\ ${ }^{1}$ Fresenius Kabi Deutschland GmbH, Else-Kröner-Strasse 1, 61352 Bad Homburg, Germany \\ ${ }^{2}$ Biocrates Life Sciences AG, Eduard-Bodem-Gasse 8, 6020 Innsbruck, Austria \\ ${ }^{3}$ Charles River Laboratories, 334 South St, Shrewsbury, MA 01545, USA \\ ${ }^{4}$ Charles River Laboratories, Bois des Oncins, 69210 Saint-Germain-Nuelles, France
}

(Received July 27, 2017; Accepted September 15, 2017)

\begin{abstract}
Plasma amino acid level changes occur in mild, moderate and severe stages of liver injury in human patients. In animal models, however, data are mainly restricted to severe liver injury models in rats. Here we present the characterization of a rat model of moderate liver dysfunction secondary to alpha-napthylisothiocyanate (ANIT)-induced cholestasis. Rats treated with $30 \mathrm{mg} / \mathrm{kg} / \mathrm{day}$ ANIT for 3 weeks exhibited a time-dependent increase in plasma alanine aminotransferase (ALT), aspartate aminotransferase (AST) and bilirubin levels and a decrease in albumin concentration. According to a liver dysfunction evaluation based on the human Child-Pugh-Score, animals developed a moderate liver dysfunction in the first two weeks of ANIT treatment, while only a mild dysfunction was observed at the end of week 3 despite ongoing ANIT administration. Univariate analysis of branched-chain amino acid plasma levels indicated that reduced levels of branched chain amino acids were associated with the ANIT treatment. These data may set the stage for further research of amino acid disturbances and requirements in non-severe cholestasis.
\end{abstract}

Key words: Animal model, Liver dysfunction, Cholestasis, Alpha-naphthylisothiocyanate

\section{INTRODUCTION}

The liver is one of the main organs for amino acid metabolism and plasma amino acid disturbances due to severe liver injury have been associated with patient outcome in end-stage liver injury (Kinny-Köster et al., 2016). Such amino acid disturbances are obviously not limited to severe liver injury only, they also occur in mild or moderate liver disease as for example children with mild-tomoderate cholestasis show increased requirements of branched-chain amino acids (Mager et al., 2006). The extensive analysis of the amino acid requirements in humans is, however, restricted to plasma levels of amino acids in human patients, which may not automatically reflect the situation in organs like liver or muscle. The generation of such organ level data is of course limited to animal models. To date, most available animal models focus on severe cholestasis like in the bile duct ligation model (Rodriguez-Garay, 2003) and are therefore not useful to study the milder stage of disease. Thus the aim of this study was to establish a model of mild or moderate liver injury based on the toxicant alpha-naphthylisothiocyanate (ANIT), which induces a dose dependent hepatotoxic effect based on cholestasis. Among cholestasis inducing toxicants ANIT was chosen as a starting point for the establishment of the liver dysfunction score because it is one of the most extensively studied cholestasis inducing toxicants and a well-characterized cholestasis model (Chisholm and Dolphin, 1996; Glaser et al., 2010; Lesage et al., 2001).

In patients, the grading of liver injury is usually either based on the Child-Pugh-Score (Brown, 2000) or the newer Model-of-End-Stage-Liver-Disease-(MELD)Score. The MELD-Score is based on the plasma creatinine and bilirubin levels as well as the international normalized ratio (INR), and the Child-Pugh-Score is derived from plasma levels of bilirubin, albumin, prothrombin time, occurrence of ascites as well as occurrence of hepatic encephalopathy (Peng et al., 2016). For our study, we based the grading of the liver dysfunction in an animal

Correspondence: Melanie K. Bothe (E-mail: melanie.bothe@fresenius-kabi.com) 
model on the parameters used in the Child-Pugh-Score, because this score distinguishes stepwise between three grades of liver dysfunction and we aimed at a clinical grading of mild, moderate or severe liver dysfunction.

We tested the applicability of the new defined liver dysfunction score for a model of ANIT-induced liver injury and determined the plasma amino acid levels of branched-chain amino acids (BCAA) to confirm the amino acid changes observed in mild-to-moderate cholestasis in human patients.

\section{MATERIALS AND METHODS}

\section{Chemicals}

Alpha-naphthyl isothiocyanate of $99.1 \%$ purity was obtained from Sigma-Aldrich (Saint Louis, MO, USA).

\section{Animals}

Twenty Wistar: Crl: WI (Han) rats were obtained from Charles River Laboratories (Domain des Oncins, France) and had an acclimatization period of at least seven days before start of the experiments at the age of 11 weeks. All animals were housed in groups of five in a temperature controlled room with a 12-hr light/dark cycle. The room temperature and relative humidity were maintained in the ranges of $22 \pm 3{ }^{\circ} \mathrm{C}$ and $>35 \%$. All animals had access to food (diet C 1003 modified to contain 24\% protein) obtained from Altromin (Lage, Germany) and water ad libitum. The body weights at the start of the experiments ranged from 304 to $333 \mathrm{~g}$. The study was approved by the ethical committee of Charles River and authorized by the French authorities.

\section{Experimental design}

The experiment was conducted in 2 phases. The first phase aimed at the characterization of the basic parameters to calculate the liver dysfunction grade of the animals, while in the second phase the plasma amino acids of animals treated with an ANIT dose determined to induce a moderate liver dysfunction were analyzed. For each phase, animals were divided into groups of $n=5$. Animals in the treatment group received 20,30 , or $40 \mathrm{mg} / \mathrm{kg} /$ day ANIT via single daily oral gavage for 21 days (phase 1) or $30 \mathrm{mg} / \mathrm{kg} /$ day for 16 days (phase 2); animals in the control group received the vehicle corn oil. In phase 1 the treatment with $20 \mathrm{mg} / \mathrm{kg} /$ day and $40 \mathrm{mg} / \mathrm{kg} /$ day was performed for 7 days only. The $20 \mathrm{mg} / \mathrm{kg}$ /day group was stopped because this dose did not lead to an increase in the liver dysfunction score after one week (Supplemental Table 1), and the $40 \mathrm{mg} / \mathrm{kg} /$ day group was stopped due to the provisions of the ethical committee of the test facility because this dose led to more than $10 \%$ decrease in body weight. Treatment with $30 \mathrm{mg} / \mathrm{kg} /$ day and vehicle lasted for 21 days in phase 1 and for 16 days in phase 2 .

\section{Blood collection}

Blood was taken from the tail vein (amino acids, ammonia, and prothrombin time) as well as the retroorbital sinus under isoflurane anesthesia (clinical chemistry parameters). In phase 1 , blood samples were taken before and on days 7, 14, and 21 or 22 after start of treatment, while in phase 2, blood sampling for plasma amino acid analysis was performed on days 7,9 and 16 after start of treatment. The sampling days in phase 2 were chosen according to the results obtained for body weight in phase 1 , day 7 represents a day during decreasing body weight, day 9 is the first day of the period with stable body weight and day 16 is one week later.

\section{Analysis of serum clinical chemistry, ammonia and prothrombin time}

Total bilirubin, albumin, aspartate aminotransferase (AST), alanine aminotransferase (ALT), and alkaline phosphatase (ALP) were determined using the AU640 Beckmann coulter system (Roissy, France). Blood ammonia was measured with the PocketChem BA PA-4140 obtained from Axonlab (Stuttgart, Germany) and prothrombin time was measured with the CoaguCheck XS obtained from Roche (Mannheim, Germany) according to the manufacturer's instruction.

\section{Calculation of the liver dysfunction score}

The liver dysfunction score was defined as depicted and compared to the human Child-Pugh-Score (CPS, (Brown, 2000)) in Table 1. Reference ranges were taken from historical control data of Charles River France or from control groups. Of note, this score can only be applied in animals where the liver enzyme ALT is already out of reference range ( $>$ mean of historical control data of Charles River France $\pm 2 \mathrm{SD}$, namely $>48 \mathrm{IU} / \mathrm{L}$ ) to ensure liver injury. This is important because the liver dysfunction grade of 4 points ("mild") represents the status of healthy rats except for increased ALT levels.

The CPS grades in humans are A (5-6 points), B (7-9 points) and $C$ (10-15 points). Ascites did not occur in our pilot trial despite the use of higher dosages of ANIT and ascites was not reported in any of the former publications with ANIT in rats. Therefore, the parameter ascites was excluded from the determination of the liver dysfunction score in this model. The maximum achievable number was then 12 for the rat score instead of 15 for the human score. The modified rat liver dysfunction grades were thus 
Characterization of a moderate liver dysfunction rat model

Table 1. Comparison of human Child-Pugh-Score and rat liver dysfunction score.

\begin{tabular}{|c|c|c|c|c|c|c|c|c|}
\hline \multirow[t]{2}{*}{ Parameter } & \multicolumn{4}{|c|}{ Human Child-Pugh-Score } & \multicolumn{4}{|c|}{ Rat Liver Dysfunction Score } \\
\hline & Ref. range & 1 point & 2 points & 3 points & Ref. range & 1 point & 2 points & 3 points \\
\hline Total serum bilirubin $(\mu \mathrm{M})$ & $2-19$ & $<68^{\mathrm{a}, \mathrm{d}}$ & $68-170^{a}$ & $>170^{\mathrm{a}, \mathrm{e}}$ & $1.31-2.58^{\mathrm{b}}$ & $<9^{\mathrm{d}}$ & $9-23$ & $>23^{\mathrm{e}}$ \\
\hline Serum albumin $(\mathrm{g} / \mathrm{L})$ & $35-54$ & $>35$ & $28-35$ & $<28$ & $30-36^{b}$ & $>30$ & $28-30$ & $<28$ \\
\hline $\begin{array}{l}\text { Prothrombin time } \\
\text { prolongation (sec) }\end{array}$ & $\begin{array}{c}12-13 \\
\text { prothrombin } \\
\text { time }\end{array}$ & $<4$ & $4-6$ & $>6$ & $\begin{array}{c}12.5-15.7^{\mathrm{b}} \\
\text { prothrombin } \\
\text { time }\end{array}$ & $<4$ & $4-6$ & $>6$ \\
\hline $\begin{array}{l}\text { Hepatic encephalopathy/ } \\
\text { Ammonia blood levels }(\mu \mathrm{M})^{\mathrm{c}}\end{array}$ & none & none & minimal & coma & $\begin{array}{c}\text { control group } \\
\text { mean } \\
\pm \text { S.D. }\end{array}$ & in ref. range & $\begin{array}{l}\text { out of ref. } \\
\text { range }\end{array}$ & coma \\
\hline Ascites & absent & absent & controlled & refractory & \multicolumn{4}{|c|}{ excluded from score } \\
\hline
\end{tabular}

: These values are already adapted for the specific liver injury of cholangitis, for which different cut off values are commonly used.

b: historical control data

c: In rats, hepatic encephalopathy occurs when blood ammonia increases. Therefore, the increase in blood ammonia was measured as substitute for encephalopathy.

$\mathrm{d}$ : $3.5 \mathrm{x}$ upper limit ref. range

e: 9x upper limit ref. range

mild (4-5 points), moderate (6-7 points) and severe (8-12 points).

\section{Analysis of amino acids}

Branched-chain amino acids were determined using the LC-MS/MS based AbsoluteIDQ ${ }^{\circledR}$ p180 Kit (Biocrates, Innsbruck, Austria) according to the manufacturer's instructions.

\section{Histopathological analysis}

At the end of phase 1, animals were euthanized by carbon dioxide inhalation and exsanguination and submitted to necropsy. The liver of each animal was fixed in $10 \%$ neutral formalin and hematoxylin \& eosin stained slides were analyzed histopathologically.

\section{Statistics}

Pairwise comparison of the treatment group and the control group was performed using the two sample t-test.

\section{RESULTS}

\section{Body weight}

Animals of the $30 \mathrm{mg} / \mathrm{kg} /$ day treatment group showed a reduction in body weight compared to the control group from day 3 to day 8 as depicted in Fig. 1A. The body weight of the treatment group remained stable from day 8 to day 19, while the control group showed an increase in body weight as expected. Statistical significant differences in body weight were observed from day 5 to day 22 $(\mathrm{p}<0.05)$. The body weight gain of the control group was statistically significantly higher at the intervals day 1 to 3 , day 3 to 5 , day 5 to 8 as well as day 12 to 15 , day 15 to 17, and day 19 to 22 as depicted in Fig. 1B ( $<<0.05)$.

\section{Serum clinical chemistry}

The ALT plasma levels of the ANIT-treated animals were most prominently elevated on day 7 and returned almost back to normal during the course of the treatment. The AST and ALP plasma levels of the ANIT-treated animals followed a similar pattern. The plasma levels of bilirubin, albumin, and ammonia as well as the prothrombin time were used to calculate the grade of liver dysfunction. During the treatment period, bilirubin plasma levels increased most prominently on day 7 and then decreased, while in contrast, albumin levels were most prominently decreased on day 7 and then returned to almost normal again. The prothrombin time was not changed as were the ammonia plasma levels, although the variability of ammonia plasma levels was quite high. The mean levels of these results are depicted in Table 2.

\section{Liver dysfunction score}

The individual liver dysfunction scores of animals treated with $30 \mathrm{mg} / \mathrm{kg} /$ day ANIT are shown in Table 3 and the median liver dysfunction grade of the animals is shown in Fig. 2. The animals treated with $30 \mathrm{mg} / \mathrm{kg} /$ day ANIT showed a median liver dysfunction score of 7 (grade moderate) on day 7, 6 (grade moderate) on day 14 and returned to 5 (grade mild) on day 22 .

\section{Histopathology}

ANIT-related microscopic findings were noted in the liver of the $30 \mathrm{mg} / \mathrm{kg} /$ day group from Phase 1 . These consisted of slight (in 1/5 animals) or moderate (in 4/5 animals) bile duct hyperplasia associated with minimal periportal subacute inflammatory cell infiltration. Bile duct hyperplasia of this severity was considered to be related to treatment and is consistent with findings reported by 
A)

\section{Bodyweight}

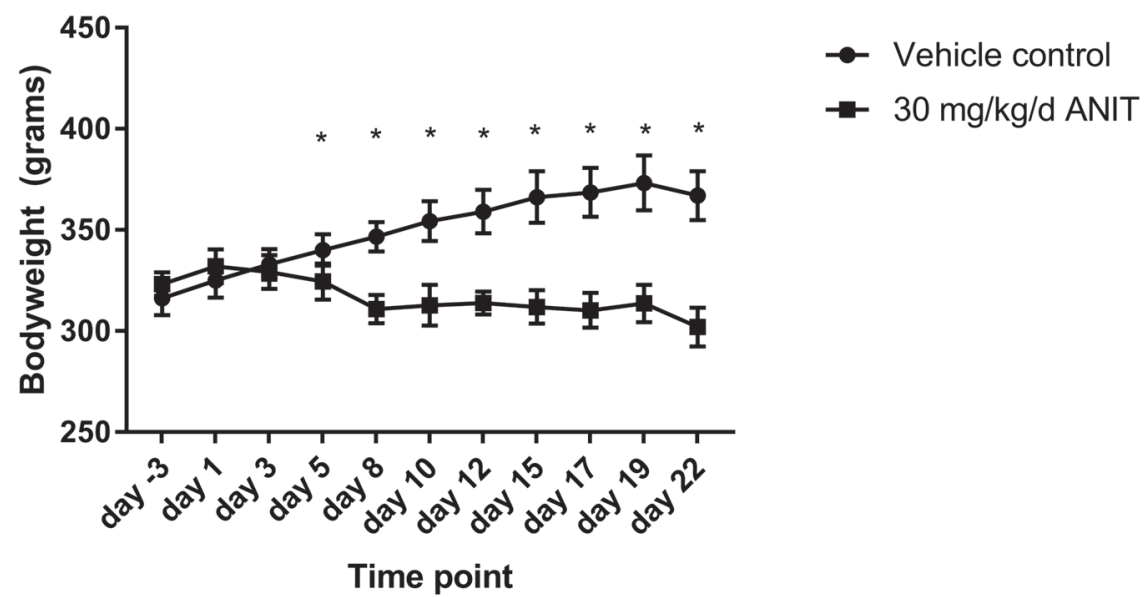

B)

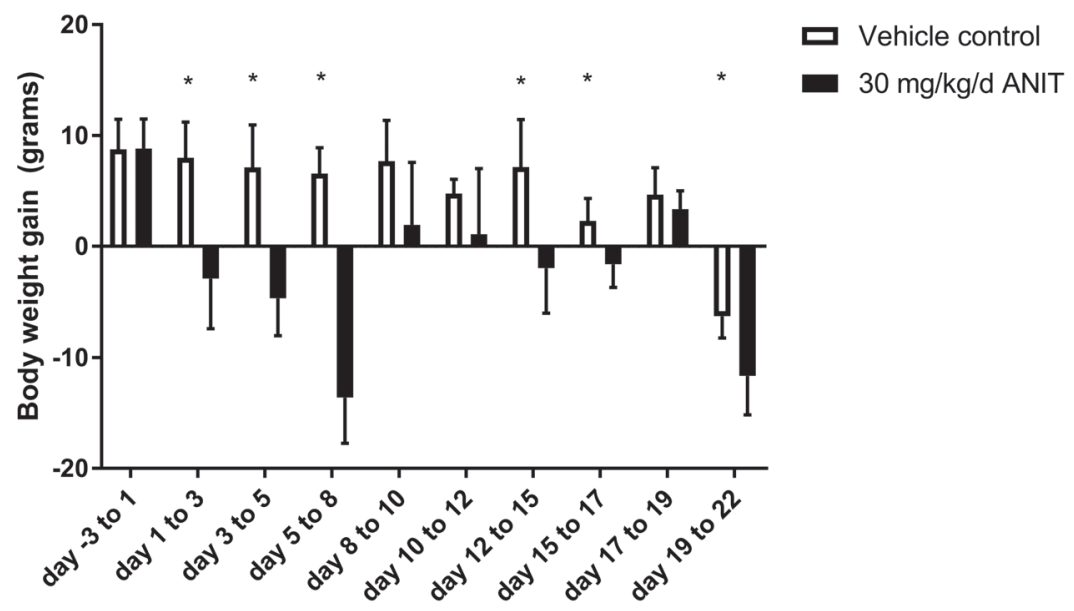

Fig. 1. Body weight and body weight gain of $30 \mathrm{mg} / \mathrm{kg} /$ day ANIT treated group and control group. 1A) Body weight decreases in the ANIT treated group from ANIT administration day 1 to day 8 and then stabilizes until sacrifice on day 22 . The body weight in the control group was statistically significantly higher from day 5 to day $22(* \mathrm{p}<0.05)$. 1B) Comparison of body weight gain of untreated animals and animals receiving ANIT again depicts the decrease in body weight gain mainly in the first 8 days of treatment. Data of day 19 to 22 are confounded by the fact that the animals were fasted the night before day 22 and are therefore diagnostically less conclusive. ${ }^{*} p<0.05$.

Goldfarb (Goldfarb et al., 1962).

\section{Branched-chain amino acids}

The plasma levels of the branched-chain amino acids were reduced throughout the ANIT administration period as depicted in Table 4. The plasma levels of leucine, isoleucine and valine were statistically significantly decreased on day 7 .

\section{DISCUSSION}

The most important result of the current study is the applicability of the newly established liver dysfunction score in a rat model of moderate cholestasis. In our hands, $30 \mathrm{mg} / \mathrm{kg} /$ day of the liver toxicant ANIT for three weeks led to an increase in plasma ALT, AST, ALP, and bilirubin as well as a decrease in albumin most prominent in the first week. The symptoms returned back to almost normal until the end of week 3 . 
Characterization of a moderate liver dysfunction rat model

Table 2. Mean values \pm S.D. of parameters used to calculate the liver dysfunction score as well as alanine aminotransferase (ALT), aspartate aminotransferase (AST), and alkaline phosphatase (ALP) plasma levels.

\begin{tabular}{|c|c|c|c|c|c|c|c|c|}
\hline \multirow{2}{*}{ Parameter } & \multicolumn{4}{|c|}{ Control group } & \multicolumn{4}{|c|}{$30 \mathrm{mg} / \mathrm{kg} /$ day ANIT group } \\
\hline & Day -3 & Day 7 & Day 14 & Day 21 & Day -3 & Day 7 & Day 14 & Day 21 \\
\hline $\begin{array}{l}\text { Total serum } \\
\text { bilirubin }(\mu \mathrm{M})\end{array}$ & $1.08 \pm 0.23$ & $1.58 \pm 0.40$ & $1.70 \pm 0.25$ & $2.32 \pm 0.08$ & $0.72 \pm 0.13^{*}$ & $96.44 \pm 18.34 * *$ & $48.14 \pm 31.53^{* *}$ & $16.92 \pm 11.33 * *$ \\
\hline $\begin{array}{l}\text { Serum albumin } \\
(g / L)\end{array}$ & $32.52 \pm 0.40$ & $32.30 \pm 0.63$ & $34.34 \pm 0.96$ & $34.40 \pm 0.50$ & $31.94 \pm 1.02$ & $28.90 \pm 0.73^{* *}$ & $32.04 \pm 1.33^{*}$ & $33.10 \pm 1.47$ \\
\hline $\begin{array}{l}\text { Prothrombin time } \\
(\mathrm{sec})\end{array}$ & $11.90 \pm 0.44$ & $12.08 \pm 0.25$ & $11.76 \pm 0.89$ & $12.44 \pm 0.45$ & $11.96 \pm 0.13$ & $11.78 \pm 0.24$ & $11.62 \pm 0.47$ & $12.58 \pm 0.51$ \\
\hline $\begin{array}{l}\text { Blood ammonia } \\
(\mu \mathrm{M})\end{array}$ & $38.6 \pm 35.2$ & $121.5 \pm 56.0$ & $119.0 \pm 31.8$ & $60.6 \pm 69.5$ & $45.0 \pm 52.1$ & $130.2 \pm 57.8$ & $73.8 \pm 41.3$ & $89.2 \pm 68.7$ \\
\hline $\begin{array}{l}\text { ALT } \\
(\mathrm{IU} / \mathrm{L})\end{array}$ & nd & $31.6 \pm 5.3$ & $34.2 \pm 8.5$ & $35.4 \pm 11.0$ & nd & $162.6 \pm 43.8^{* *}$ & $115.8 \pm 61.8^{* *}$ & $68.0 \pm 22.0$ \\
\hline $\begin{array}{l}\text { AST } \\
(\mathrm{IU} / \mathrm{L})\end{array}$ & nd & $101.8 \pm 9.6$ & $114.6 \pm 41.4$ & $101.6 \pm 8.3$ & nd & $387.2 \pm 86.1^{* *}$ & $262.8 \pm 128.6^{*}$ & $117.2 \pm 21.6$ \\
\hline $\begin{array}{l}\text { ALP } \\
(\mathrm{IU} / \mathrm{L}) \\
\end{array}$ & nd & $581.2 \pm 188.3$ & $567.8 \pm 194.9$ & $302.6 \pm 93.8$ & nd & $908.8 \pm 91.6^{*}$ & $877.0 \pm 176.1 *$ & $421.6 \pm 63.6$ \\
\hline
\end{tabular}

nd $=$ not determined. $* \mathrm{p}<0.05 . * * \mathrm{p}<0.01$

Table 3. Individual liver dysfunction scores of animals treated with $30 \mathrm{mg} / \mathrm{kg} /$ day ANIT.

\begin{tabular}{|c|c|c|c|c|c|c|c|}
\hline Animal no. & Ammonia & Prothrombin time & Bilirubin & Albumin & Total score & Median score & ALT (IU/L) \\
\hline \multicolumn{8}{|l|}{ Day 7} \\
\hline 1 & 1 & 1 & 3 & 2 & 7 & & 141 \\
\hline 2 & 1 & 1 & 3 & 2 & 7 & & 160 \\
\hline 3 & 2 & 1 & 3 & 2 & 8 & 7 & 106 \\
\hline 4 & 1 & 1 & 3 & 2 & 7 & & 222 \\
\hline 5 & 2 & 1 & 3 & 2 & 8 & & 184 \\
\hline \multicolumn{8}{|l|}{ Day 14} \\
\hline 1 & 1 & 1 & 3 & 2 & 7 & & 72 \\
\hline 2 & 1 & 1 & 3 & 1 & 6 & & 197 \\
\hline 3 & 2 & 1 & 2 & 1 & 6 & 6 & 75 \\
\hline 4 & 1 & 1 & 3 & 1 & 6 & & 168 \\
\hline 5 & 1 & 1 & 2 & 1 & 5 & & 67 \\
\hline \multicolumn{8}{|l|}{ Day 22} \\
\hline 1 & 1 & 1 & 2 & 1 & 5 & & 57 \\
\hline 2 & 1 & 1 & 2 & 1 & 5 & & 49 \\
\hline 3 & 1 & 1 & 2 & 1 & 5 & 5 & 53 \\
\hline 4 & 2 & 1 & 3 & 1 & 7 & & 101 \\
\hline 5 & 2 & 1 & 2 & 1 & 6 & & 80 \\
\hline
\end{tabular}

Cholestasis leads to secondary hepatocyte injury as reflected by increased plasma levels of liver enzymes like ALT. Hepatocyte impairment is known to lead to reduced levels of branched-chain amino acids for example in cirrhotic patients (Ouchi et al., 1989) and in patients with mild-to-moderate cholestasis as well [2]. In our model we find reduced plasma levels of BCAA throughout the period of administration, showing that similar to humans even moderate liver dysfunction impacts the BCAA metabolism. These results show that even though no hepatocyte injury was observed in the histopathological analysis at the end of the study, which is most probably due to the regeneration capacity of the liver and the transient nature of the model, hepatocytes were injured during the ANIT-treatment. According to our liver dysfunction score, this injury was moderate in nature for the first two weeks of treatment and then returned to mild/normal. The decrease in the liver dysfunction score over time is most probably due to the partial resistance of the regenerating liver to hepatotoxicants (Horiuchi et al., 1978; Roberts et al., 1983; Uryvaeva and Faktor, 1976a, 1976b) on the one hand and the induction of bile efflux transporters by ANIT leading to increased ANIT clearance on the other hand (Tanaka et al., 2009). 
Table 4. Mean values of branched-chain amino acid plasma levels.

\begin{tabular}{|c|c|c|c|c|c|c|}
\hline \multirow{2}{*}{ Parameter $(\mu \mathrm{M})$} & \multicolumn{3}{|c|}{ Control group } & \multicolumn{3}{|c|}{ ANIT group } \\
\hline & Day 7 & Day 9 & Day 16 & Day 7 & Day 9 & Day 16 \\
\hline \multicolumn{7}{|l|}{ Amino acids } \\
\hline Isoleucine & $164.0 \pm 12.1$ & $180.2 \pm 40.8$ & $171.1 \pm 59.3$ & $119.8 \pm 35.1 *$ & $145.8 \pm 23.5$ & $153.7 \pm 37.9$ \\
\hline Leucine & $286.4 \pm 27.6$ & $312.4 \pm 90.6$ & $304.6 \pm 123.0$ & $205.8 \pm 72.7^{*}$ & $258.0 \pm 41.7$ & $268.4 \pm 72.1$ \\
\hline Valine & $379.0 \pm 46.6$ & $386.4 \pm 66.4$ & $370.6 \pm 143.3$ & $275.8 \pm 87.6^{*}$ & $351.6 \pm 56.2$ & $348.6 \pm 88.8$ \\
\hline
\end{tabular}

$* \mathrm{p}<0.05$

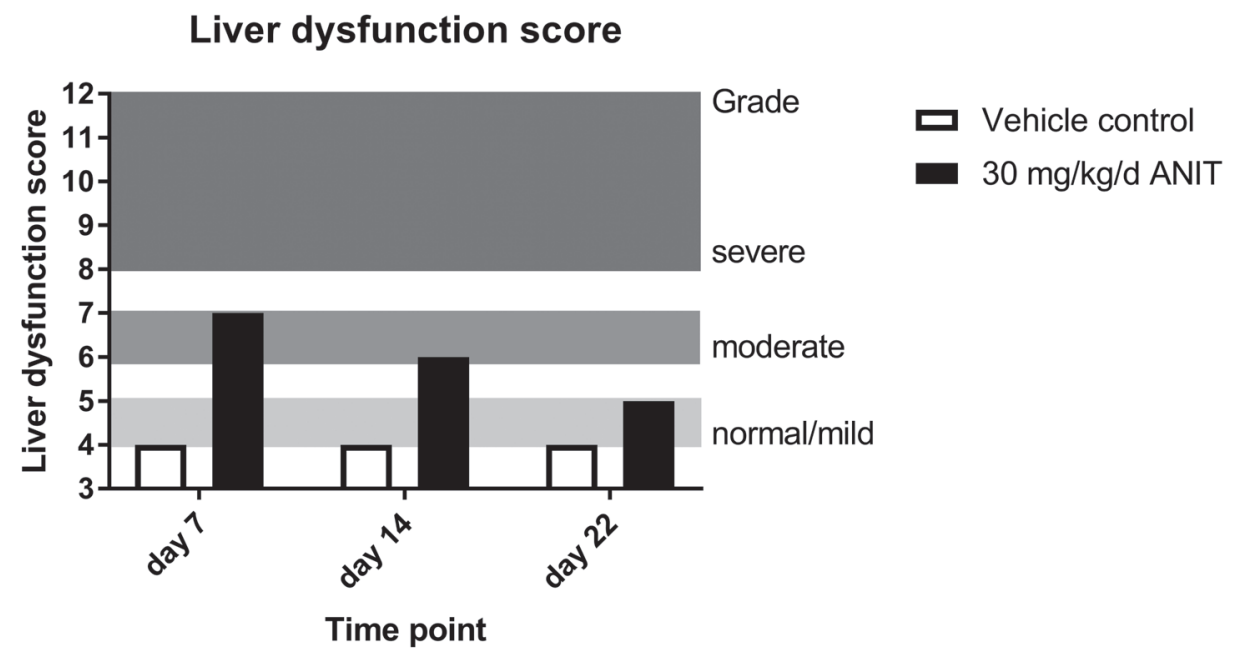

Fig. 2. Liver dysfunction score of $30 \mathrm{mg} / \mathrm{kg}$ /day ANIT treated animals at days 7,14 , and 22 of treatment. The median liver dysfunction score depicted here shows that ANIT-induced cholestasis leads to moderate liver dysfunction on day 7 and day 14 of ANIT treatment, while on day 22 the liver dysfunction is in the mild/normal range.

In recent studies, ANIT was mainly applied in high dosages via the oral or the intraperitoneal route (Aoki et al., 1986; Leonard et al., 1984; Saitoh et al., 2014) either as a single or a repeated administration. ANIT is known to dose-dependently increase the plasma levels of bilirubin, GGT and bile acids and to transiently increase the levels of ALT (Leonard et al., 1984), starting at a dose of $0.01 \%$ ANIT in the regular diet. Oral single dosages of $60-100 \mathrm{mg} / \mathrm{kg}$ ANIT, which are known to lead to severe liver injury depicted by more than fourfold increase in ALT, AST, and bilirubin levels, induce amino acid disturbances in the rat (Aoki et al., 1986; Saitoh et al., 2014). None of these former studies, however, characterized the chronic administration of a distinctive dose of ANIT according to a liver dysfunction score and then investigated the amino acid plasma levels in a model of moderate liver dysfunction.

A limitation of our study is the increased adaptation and resistance of the liver to the toxicant ANIT over time. A similar time course is not expected for example in cholestatic patients, therefore the branched-chain ami- no acid plasma levels measured two weeks after start of ANIT-treatment during the period of adaptation and regeneration have to be interpreted carefully. Furthermore, in this paper we rely on plasma amino acid levels as a starting point, which are admittedly comparable to the observations in patients, however, the main advantage of this established animal model would be the chance to investigate organ amino acid levels as well in the future. Next steps should also include investigating the applicability of our liver dysfunction score to cholestasis induced by other toxicants and to other models of liver injury.

Taken together, we present for the first time a model of moderate liver dysfunction resulting from ANIT-induced cholestasis in rats. Plasma branched-chain amino acid reductions similar to human patients were found and these results may provide the basis for further research of amino acid requirements in this model of moderate liver dysfunction.

Conflict of interest---- MKB, CM, JH, and MW are employees of Fresenius Kabi Deutschland GmbH, the 
Characterization of a moderate liver dysfunction rat model

sponsor of this study. UM is employee of Biocrates Lifescience AG, VR and JCQ are employees of Charles River.

\section{REFERENCES}

Aoki, Y., Suzuki, H. and Itoh, H. (1986): Changes in polyamine metabolism in rat liver after oral administration of alpha-naphthylisothiocyanate. J. Toxicol. Sci., 11, 95-104.

Brown, R.S.Jr. (2000): Predicting outcome of transplantation for cholestatic liver disease: Child-Pugh or Mayo risk score? Liver Transpl., 6, 759-761.

Chisholm, J.W. and Dolphin, P.J. (1996): Abnormal lipoproteins in the ANIT-treated rat: a transient and reversible animal model of intrahepatic cholestasis. J. Lipid. Res., 37, 1086-1098.

Glaser, S.S., Onori, P., Wise, C., Yang, F., Marzioni, M., Alvaro, D., Franchitto, A., Mancinelli, R., Alpini, G., Munshi, M.K. and Gaudio, E. (2010): Recent advances in the regulation of cholangiocyte proliferation and function during extrahepatic cholestasis. Dig. Liver Dis., 42, 245-252.

Goldfarb, S., Singer, E.J. and Popper, H. (1962): Experimental cholangitis due to alpha-naphthyl-isothiocyanate (ANIT). Am. J. Pathol., 40, 685-698.

Horiuchi, T., Ohtsubo, K. and Saito, M. (1978): Development of resistance to hepatotoxic effect of furylfuramide by pretreatment with its subnecrotic doses and carbon tetrachloride. Jpn. J. Exp. Med., 48, 27-33.

Kinny-Köster, B., Bartels, M., Becker, S., Scholz, M., Thiery, J., Ceglarek, U. and Kaiser, T. (2016): Plasma Amino Acid Concentrations Predict Mortality in Patients with End-Stage Liver Disease. PloS One, 11, e0159205.

Leonard, T.B., Neptun, D.A. and Popp, J.A. (1984): Serum gamma glutamyl transferase as a specific indicator of bile duct lesions in the rat liver. Am. J. Pathol., 116, 262-269.

Lesage, G., Glaser, S., Ueno, Y., Alvaro, D., Baiocchi, L., Kanno,
N., Phinizy, J.L., Francis, H. and Alpini, G. (2001): Regression of cholangiocyte proliferation after cessation of ANIT feeding is coupled with increased apoptosis. Am. J. Physiol. Gastrointest. Liver Physiol., 281, G182-190.

Mager, D.R., Wykes, L.J., Roberts, E.A., Ball, R.O. and Pencharz, P.B. (2006): Branched-chain amino acid needs in children with mild-to-moderate chronic cholestatic liver disease. J. Nutr., 136, 133-139.

Ouchi, K., Matsubara, S., Fukuhara, K. and Matsuno, S. (1989): Plasma amino acid abnormalities in liver disease: comparative analysis of idiopathic portal hypertension, extrahepatic portal occlusion and liver cirrhosis. Tohoku J. Exp. Med., 158, 171178.

Peng, Y., Qi, X. and Guo, X. (2016): Child-Pugh Versus MELD Score for the Assessment of Prognosis in Liver Cirrhosis: A Systematic Review and Meta-Analysis of Observational Studies. Medicine, 95, e2877.

Roberts, E., Ahluwalia, M.B., Lee, G., Chan, C., Sarma, D.S. and Farber, E. (1983): Resistance to hepatotoxins acquired by hepatocytes during liver regeneration. Cancer Res., 43, 28-34.

Rodriguez-Garay, E.A. (2003): Cholestasis: human disease and experimental animal models. Ann. Hepatol., 2, 150-158.

Saitoh, W., Yamauchi, S., Watanabe, K., Takasaki, W. and Mori, K. (2014): Metabolomic analysis of arginine metabolism in acute hepatic injury in rats. J. Toxicol. Sci., 39, 41-50.

Tanaka, Y., Aleksunes, L.M., Cui, Y.J. and Klaassen, C.D. (2009): ANIT-induced intrahepatic cholestasis alters hepatobiliary transporter expression via $\mathrm{Nrf2}$-dependent and independent signaling. Toxicol. Sci., 108, 247-257.

Uryvaeva, I.V. and Faktor, V.M. (1976a): [Relationship between cell division and function. Resistance of the liver to toxic effect of CCL4 after partial hepatectomy]. Tsitologiia, 18, 1354-1359.

Uryvaeva, I.V. and Faktor, V.M. (1976b): [Resistance of regenerating liver to hepatotoxins]. Biull. Eksp. Biol. Med., 81, 283-285. 